\title{
ARTICLE \\ Genotyping-by-sequencing of pear (Pyrus spp.) accessions unravels novel patterns of genetic diversity and selection footprints
}

\author{
Satish Kumar ${ }^{1}$, Chris Kirk ${ }^{2}$, Cecilia Deng ${ }^{3}$, Claudia Wiedow ${ }^{2}$, Mareike Knaebel ${ }^{2}$ and Lester Brewer ${ }^{4}$
}

Understanding of genetic diversity and marker-trait relationships in pears (Pyrus spp.) forms an important part of gene conservation and cultivar breeding. Accessions of Asian and European pear species, and interspecific hybrids were planted in a common garden experiment. Genotyping-by-sequencing (GBS) was used to genotype 214 accessions, which were also phenotyped for fruit quality traits. A combination of selection scans and association analyses were used to identify signatures of selection. Patterns of genetic diversity, population structure and introgression were also investigated. About 15000 high-quality SNP markers were identified from the GBS data, of which $25 \%$ and $11 \%$ harboured private alleles for European and Asian species, respectively. Bayesian clustering analysis suggested negligible gene flow, resulting in highly significant population differentiation $\left(F_{s t}=0.45\right)$ between Asian and European pears. Interspecific hybrids displayed an average of 55\% and $45 \%$ introgression from their Asian and European ancestors, respectively. Phenotypic (firmness, acidity, shape and so on) variation between accessions was significantly associated with genetic differentiation. Allele frequencies at large-effect SNP loci were significantly different between genetic groups, suggesting footprints of directional selection. Selection scan analyses identified over 20 outlier SNP loci with substantial statistical support, likely to be subject to directional selection or closely linked to loci under selection.

Horticulture Research (2017) 4, 17015; doi:10.1038/hortres.2017.15; Published online 12 April 2017

\section{INTRODUCTION}

Pear belongs to the genus Pyrus in the family Rosaceae, and has a basic chromosome number of $x=17$. The number of catalogued species, most of which are diploid $(2 n=34)$, in the genus Pyrus vary according to different studies, but there could be as many as 75 species. ${ }^{1}$ It is believed that genus Pyrus originated during the Tertiary period ( 65 to 55 million years ago) in the mountainous regions of western China. Evidence suggests that pear dispersion and speciation followed the mountain ranges to both the east and the west. ${ }^{2}$ The ancient Romans made a great contribution to pear domestication by developing methods of propagation, grafting and caring for fruit. There were reported to be more than 40 cultivars existing in the 1 st century B.C. ${ }^{1}$ Pear has been cultivated for at least 2000-3000 years, and is currently grown commercially in $>50$ countries in Europe, Northern Africa, Asia, Australasia and North America. ${ }^{3}$ One of the main reasons breeding programmes are present in almost every continent is because it is important to have cultivars adapted to their growing environment. In spite of the wide geographical distribution of the genus, there are no major incompatibility barriers to interspecific hybridisation. Interspecies hybrids are sometimes developed in pear breeding programmes to produce new cultivars with novel combinations of texture and flavour, and to improve resistance to pests and diseases. $^{4,5}$

Molecular markers have become the preferred tools for characterising genetic diversity. The most frequently used method to assess population differentiation is the calculation of $F_{s t}$ a summary statistic that quantifies the variation in marker allele frequencies between populations. ${ }^{6}$ Genetic diversity and genetic relatedness studies within and between species in Asian pears identified markers specific to species, and the clustering of species was largely in agreement with their geographic distribution. ${ }^{7-9}$ Genetic analysis of 145 wild and cultivated accessions of $P$. communis clearly separated accessions native to the Caucasus Mountains from those native to Eastern European countries. ${ }^{10}$ Clustering patterns corresponding with geographic origin were also observed among $P$. communis accessions collected from 12 provenances in Northern Spain. ${ }^{11}$

Studies on genetic diversity among Asiatic and European pears revealed three genetic groups, with the primary division between occidental (Europe and Central Asia) and oriental (East Asia) pears, followed by division of Japanese and Chinese accessions. ${ }^{7,12,13}$ Artificial as well as natural interspecific hybridisation have resulted in complex population structures of pear accessions. Bayesian inference of population structures showed that Japanese $P$. ussuriensis was genetically admixed with two genetic clusters: true native $P$. ussuriensis var. ussuriensis and prehistorically introduced $P$. pyrifolia. ${ }^{14}$ Clustering patterns of some $P$. communis accessions from Turkey and Macedonia indicated gene flow and introgression resulting from co-occurring congeneric subspecies. ${ }^{10}$ Some earlier studies using dominant markers revealed that the Chinese sand pear ( $P$. pyrifolia) and the white pear $\left(P . \times\right.$ bretschneideri) might share a common ancestor. ${ }^{8}$

Pyrus diversity studies to date have relied on a limited number of markers $(<150)$. Using a small number of markers can only detect genetic diversity of limited regions of the genome, and

${ }^{1}$ The New Zealand Institute for Plant \& Food Research Limited, Hawke's Bay Research Centre, Havelock North, New Zealand; ${ }^{2}$ Palmerston North Research Centre, Palmerston North, New Zealand; ${ }^{3}$ Mount Albert Research Centre, Auckland, New Zealand and ${ }^{4}$ Motueka Research Centre, Motueka, New Zealand.

Correspondence: S Kumar (Satish.Kumar@plantandfood.co.nz)

Received: 7 December 2016; Revised: 22 February 2017; Accepted: 22 February 2017 
could lead to biased or misleading inferences about $F_{\text {st. }}{ }^{15}$ Moreover, simulation ${ }^{16,17}$ and empirical ${ }^{18}$ studies have shown that SSR loci are likely to produce a significant downward bias in estimates of $F_{s t}$ because of the mutational characteristics of highly polymorphic microsatellites. Genome-wide dense genotyping of Pyrus species should offer a method of obtaining more reliable estimates of genetic diversity. Wu et al. ${ }^{19}$ published the draft genome $(512.0 \mathrm{Mb})$ of the Chinese pear cultivar 'Dangshansuli' ( $P . \times$ bretschneideri), which was followed by the publication of a draft genome $(577.3 \mathrm{Mb})$ of the European pear cultivar 'Bartlett'. ${ }^{20}$ These resources provided opportunities to develop high-density genotyping platforms such as genotyping-by-sequencing (GBS), which is a reduced representation sequencing technology (which involve digestion of genomic DNA with specific restriction enzymes) currently being used for linkage map construction, genomic selection, genome-wide association (GWA) analysis and genetic diversity studies in various plant species. ${ }^{21,22}$

When a large number of genome-wide markers are genotyped across multiple populations, empirical distribution of $F_{\text {st }}$ values can be used to identify loci that have been affected by selection. ${ }^{23}$ Such resources can also facilitate the identification of private alleles as well as genomic regions subject to distinct selective environments in geographically separated populations. The New Zealand Institute for Plant \& Food Research Limited (PFR) pear breeding programme is diverse, spanning European and Asian species as well as hybrids between these species in an attempt to create new cultivars. The main objective of this study was to conduct a GBS survey of accessions representing European and Asian pear species and interspecific hybrids, to assess genetic diversity, phylogeny and population structure. In this work, we describe the analysis of genome-wide single-nucleotide polymorphic (SNP) allele frequency differences between populations, which provides a powerful approach to interrogate the genome for signatures of selection. We also used GWA analysis to identify genomic regions underlying genetic variation in several fruit traits.

\section{MATERIAL AND METHODS}

Plant material and phenotypes

Accessions of various pear species were imported from around the world and crossing between European and Asian species and among Asian species commenced in $1983 .{ }^{24}$ In addition to the imported accessions, a large number of advanced selections made from the hybrid families are also propagated on to Quince $C$ or Quince BA29 rootstock, interstocked with 'Beurre Hardy', in PFR's Pear Repository. For the purpose of this study a total of 214 accessions, including $35 \mathrm{P}$. pyrifolia, 9 P. $\times$ bretschneideri, 1 P. pashia, 1 P. betulaefolia, 2 P. calleryana, 112 P. communis, and 54 interspecific hybrids (Supplementary Table S1) were sampled. Young leaves were collected in spring 2013 for DNA extraction.

Fruit were harvested in the fruiting season (February to May) in 2014 and 2015 when fruit background colour was beginning to change from green to yellow. Six fruit from each seedling were stored for 28 days at $3^{\circ} \mathrm{C}$, then a further 1 day at $20^{\circ} \mathrm{C}$ before evaluation. Phenotypic information on traits describing visual, sensory and instrumental fruit properties was obtained, and the six fruit were given one overall score for each trait. Briefly, skin russet coverage (RUS) and skin bitterness (BIT) were scored on scales 0 (none) to 9 (highest). Skin over-colour coverage was analysed as a presence (red)/absence (no red) trait. Scuffing (SCUF) was rated on a $0-9$ scale $(0=$ no darkening; $9=$ solid brown or black colouration) after each fruit was firmly rubbed across the cup of a moulded pulp fibreboard fruit packing tray and assessed after $2 h^{25}$ Fruit shape index was visually scored using a shape chart developed in-house (Supplementary Figure S1). Fruit firmness (FF) was determined on opposite sides of each fruit after peel removal using a Fruit Texture Analyzer (GÜSS) fitted with an 11-mm diameter probe tip. Bulked juice from the cortical flesh of the sample fruit was used to measure titratable acidity (TA) using an automatic acid titrator (Metrohm 716 DMS).

\section{Phenotypic data analysis}

Estimates of variance components for each trait were obtained using the following linear mixed model:

$$
\boldsymbol{y}=X \boldsymbol{b}+\boldsymbol{Z}_{1} \boldsymbol{a}+\boldsymbol{Z}_{2} \boldsymbol{t}+\boldsymbol{e}
$$

where $\boldsymbol{y}$ is a vector of phenotypes on a trait; $\boldsymbol{b}$ is a vector of fixed effects (that is, the intercept, year); $\mathbf{a} \sim N\left(0, \mathbf{G} \sigma_{\mathrm{a}}^{2}\right)$ is a vector of random additive effects of accessions with variance $\sigma_{a}^{2} ; \boldsymbol{G}$ is the additive relationship matrix; $\boldsymbol{t} \sim N\left(0, \boldsymbol{I}_{\mathrm{s}} \otimes \boldsymbol{G} \sigma_{\text {as }}^{2}\right)$ is a vector of random interactions of accessions $(a)$ with year $(s) ; \sigma_{\text {as }}^{2}$ represents interaction variance; $\boldsymbol{I}_{\mathrm{s}}$ represents an identity matrix with order equal to the number of years; $\otimes$ denotes the Kronecker product operation; $X, Z_{1}$ and $Z_{2}$ are incidence matrices for the fixed effects, random accession effects, and interaction effects, respectively; $\mathbf{e} \sim N\left(0, \sigma_{\mathrm{e}}^{2}\right)$ is a vector of random residual terms with variance $\sigma_{e}^{2}$. The $\boldsymbol{G}$ matrix was constructed using SNP marker information according to method from a previous study. ${ }^{26}$ ASReml software ${ }^{27}$ was used for estimation of variance components. Ratio of the additive variance $\left(\sigma_{\mathrm{a}}^{2}\right)$ to the phenotypic variance $\left(=\sigma_{\mathrm{a}}^{2}+\sigma_{\mathrm{as}}^{2}+\sigma_{\mathrm{e}}^{2}\right)$ was interpreted as heritability $\left(h^{2}\right)$.

\section{DNA extraction and GBS library preparation}

Total DNA (DNA) was extracted from leaf material after ball bearing milling (Omni Bead Rupter, Omni International), for 1 min at $3.55 \mathrm{~m} \mathrm{~s}^{-1}$ in a CTAB based buffer. ${ }^{28}$ The homogenate was incubated at $65^{\circ} \mathrm{C}$ for $30 \mathrm{~min}$, cooled and a chloroform extraction performed. The samples were centrifuged to separate the chloroform phase and insoluble plant material from the aqueous phase layer containing the DNA. This aqueous phase was pipetted into a new tube and the DNA was precipitated with the addition of $2 / 3$ vol. isopropanol and centrifuged for $10 \mathrm{~min}$ at $14000 \mathrm{~g}$. The DNA pellet was washed two times with $70 \%$ ethanol, lightly dried and finally resuspended in TE (10/0.1) buffer. The DNA concentrations were determined by fluorimetry (Qubit, Life Technologies, Waltham, MA, USA) according to manufacturer's instructions. One hundred nanograms of each DNA sample was electrophoresed $\left(3-4 \mathrm{~V} \mathrm{~cm}^{-1}\right)$ on a $1 \%(\mathrm{w} / \mathrm{v})$ agarose gel in TAE buffer, with in-gel staining using RedSafe (iNtRON Biotechnology, Korea) and UV illumination for visualisation to determine the quality level of intact, high molecular weight DNA.

GBS libraries were prepared for each DNA sample using a small modification on the protocol developed by Elshire et al. ${ }^{21}$. Ninetysix bar-code adaptors designed by Deena Bioinformatics, ${ }^{29}$ the + and - strand oligonucleotides, set out in plate format were annealed, quantified by high-sensitivity double-stranded-DNAspecific fluorimetry (Qubit) and the concentrations were normalised. A common adaptor, + and - oligonucleotides, were also annealed and quantified, and added to each bar-code adaptor well. The final working concentrations of each adapter per well was $0.3 \mathrm{ng} / \mu \mathrm{l}$. Six microlitres of this adaptor mix was pipetted to a new 96-well plate and dried down under light vacuum (CentriVap concentrator, Labconco, Kansas City, MO, USA). One hundred nanograms of DNA from a plant sample was aliquoted into a well and also dried down. The samples were re-suspended in a digest cocktail of BamHI type II restriction endonuclease (New England Biolabs, Ipswich, MA, USA) and incubated according to manufacturer's instructions. The barcoded and common adaptors were ligated to the digested DNA with a T4 DNA ligase (Promega, Madison, WI, USA) cocktail and incubated according to the manufacturer's instructions. The ligation of the adaptors to the cut ends of the DNA does not re-create the restriction enzyme site. Then $2.5 \mu \mathrm{L}$ of each library was amplified following Elshire et al. ${ }^{21}$ with primers (PPA and PPB) that annealed to sites on the adaptors 
using a high fidelity DNA polymerase (Life Technologies). After an initial denaturation step of $95^{\circ} \mathrm{C}, 2 \mathrm{~min}$, reactions were then cycled $\left(95^{\circ} \mathrm{C}, 30 \mathrm{~s} \rightarrow 65^{\circ} \mathrm{C}, 30 \mathrm{~s} \rightarrow 68^{\circ} \mathrm{C}, 30 \mathrm{~s}\right) 25$ times before a final elongation step of $68^{\circ} \mathrm{C}, 5 \mathrm{~min}$. An aliquot of each amplification was electrophoresed on a 3\% agarose gel and stained with ethidium bromide before visualisation under UV illumination to examine the library fragments. The remaining volume from each library amplification were pooled without normalisation and cleaned up on a PCR clean-up column (Qiagen, Hilden, Germany) prior to sequencing. GBS libraries were multiplexed into 5 pools, with 36 to 55 libraries per pool, for next-generation sequencing (NGS).

\section{Variants discovery from GBS data}

The sequencing was performed at Macrogen Inc., Seoul, Republic of Korea. Each pool of GBS libraries was sequenced in one lane, on the Illumina HiSeq2000 platform (San Diego, CA, USA), in singleend mode. The read length was 101 bases and the output was 165 to 203 million reads per lane. The quality of the original sequencing files was examined using FastQC (version 0.11.1; Babraham Bioinformatics; Cambridge, UK) to ensure that the data yield was acceptable and the quality was satisfactory with Phred scores $>20$ along the reads. A TASSEL ${ }^{30}$ compatible key file was constructed for all plates based on the plate layout of the GBS library preparation, the sequencing flowcell code and the sequencing lane for the plate using PERL script that was developed in-house. Five lanes of GBS data were analysed simultaneously using TASSEL/GBS pipeline on the Linux platform to discover SNP falling within 64 bases of a BamHI site. The sequencing reads were converted to TASSEL tags using the FastqToTagCount plug-in that requests at least three supporting reads for a tag. Duplicate tags from different lanes were merged with the MergeMultipleTagCount plug-in. Along with the raw sequencing data and the key file, the tags were separated according to samples using SeqToTBTHDF5 plug-in to generate tag-by-taxa (TBT) results. The merged unique tags were converted to Fastq format through TagCountToFasta plug-in.

After the tag Fastq file was constructed, two analyses were carried out, with $P . \times$ bretschneideri (cultivar 'Suli') and $P$. communis (cultivar 'Bartlett') as reference genomes, respectively. In each analysis, the Fastq file was mapped to the reference genome using Bowtie2 (version 2.2.1) ${ }^{31}$ in the 'very-sensitive-local' mode. The alignments were converted into the tag-on-physical-map (TOPM) format using the SAMConverter plug-in, which was followed by the ModifyTBTHDF5 plug-in for efficient SNP calling. On the basis of the TBT and TOPM results, SNP sites were called using the TagsToSNPByAlignment plug-in with 'minimum minor allele frequency' set to 0.001 , 'minimum minor allele count' to 3 , 'minimum locus coverage' to 0.1 and allowing rare alleles calls at site. To improve the reliability of SNP calling, filtering was applied using the GBSHapMapFilters plug-in with 'minimum site coverage' set to 0.9.

The publically available genetic map of an interspecific family, ${ }^{32}$ and a new GBS-based map of a $P$. communis family, ${ }^{33}$ were then used to assign scaffolds to different linkage groups (LG). Details describing the genetic maps were retrieved from these two papers, and converted to tab-delimited text format with columns: Marker name; Scaffold ID; Map position (cM on LG), LG; and Physical position (on scaffold). For each scaffold in the genetic maps, the number of SNP and LG identifiers were summarised using PERL script. If a scaffold had all its SNP markers mapped onto one LG in the genetic maps, the scaffold was assigned to that LG and classified as type I scaffold. If SNP on one scaffold were assigned to multiple LGs, the scaffold was classified as type II scaffold. All SNP detected on P. communis type I scaffolds (based on the Li et al..$^{33}$ genetic map) were retained. Similarly, SNP on $P . \times$ bretscheneideri type I scaffolds in the Wu et al. ${ }^{32}$ genetic map were kept for further processing. Multi-allelic SNP were discarded, along with SNP with minor allele frequency (MAF) $<0.025$, and missing data frequency $>10 \%$. Missing genotypes at the remaining SNP loci were imputed using Linklmpute software, ${ }^{34}$ which implements a $k$-nearest neighbour genotype imputation method designed for unordered markers.

Population structure and linkage disequilibrium analysis

We first constructed an un-rooted neighbour-joining (NJ) tree, which is an empirical description of a distance matrix, using the $\mathrm{R}$ package 'ape ${ }^{, 35}$ with default settings. The population structure was investigated using the model-based Bayesian clustering method implemented in STRUCTURE, ${ }^{36}$ which uses Markov Chain Monte Carlo simulations to infer the proportion of ancestry of genotypes in $K$ distinct predefined clusters. Ten independent runs were carried out for different $K$ parameter values ( $K=1$ to 4 ), and we used 50000 Markov Chain Monte Carlo iterations after a burnin of 5000 steps. Principal component analysis of the genotypic data matrix was also conducted to evaluate clustering patterns of all 214 accessions. Pairwise linkage disequilibrium (LD) between SNP markers was calculated to evaluate the extent of LD decay. The degree of LD was quantified with the parameter $r^{2}$ obtained by taking into account the population structure and cryptic relatedness using $\mathrm{R}$ software 'LDcorSV' version 1.3.1. ${ }^{37}$

\section{Evidence of selection}

Population genetic parameters including observed heterozygosity $\left(H_{\mathrm{o}}\right)$, gene diversity $\left(H_{\mathrm{s}}\right)$ and a measure of allele frequency differences between genetic groups $\left(F_{s t}\right)$ were calculated at each SNP locus, and also across all loci, using R package 'hierfstat'. ${ }^{38}$ Genome scans for outlier $F_{s t}$ values, as an evidence of selection, were also conducted using BayeScan software. ${ }^{39}$ For this purpose, $F_{\text {st }}$ coefficients are decomposed into a population-specific component (beta), shared by all loci and a locus-specific component (alpha) shared by all the populations using a logistic regression. A positive value of alpha suggests diversifying selection, whereas negative values suggest balancing or purifying selection. BayeScan estimates the probability that a locus is under selection by calculating a Bayes factor (BF), which is the ratio of the posterior probabilities of two models (selection/ neutral) given the data. A BF between 3 and $10\left(\log _{10}(B F)=0.5-\right.$ 1.0 ) is considered as a 'substantial evidence' of different statistical support for the two models. ${ }^{39}$

We also tested for genome-wide signals of marker-phenotype association to determine whether the loci of functional (associated with economically important traits) significance coincided with the outlier loci. We used the best linear unbiased estimate (BLUE), adjusted for year effect, as the phenotypic traits. ${ }^{40}$ BLUE were calculated by fitting the following fixed-effects model in ASReml software: ${ }^{27} \quad$ [phenotype $=$ mean+year+accession+(year $\times$ accession)+residuals]. Marker-trait association analyses were conducted using a mixed linear model approach implemented in GAPIT. ${ }^{41} \mathrm{~A}$ realised relationship matrix (K-matrix) and covariates from Q-matrix (derived from principal component analysis), calculated by GAPIT, were used as a correction for cryptic relatedness and population stratification, respectively, in the association models.

\section{RESULTS}

Estimates of variance components and heritability

The additive variance $\left(\sigma_{a}^{2}\right)$ was the major source of variability for all traits except TA and BIT (Table 1). On average, the additive variance accounted for $55 \%$ of the phenotypic variation. For various traits, the magnitude of genotype-by-year interaction variance varied between 0 (SCU, COL) and 29\% (Shape), with an average of $14 \%$ (Table 1). The heritability estimate $\left(h^{2}\right)$ was low $(<0.20)$ for TA, moderate $(0.20-0.40)$ for BIT, and high $(>0.40)$ for 
Table 1. Estimates of additive genetic variance $\left(\sigma_{\mathrm{a}}^{2}\right)$, genotype-by-year interaction variance $\left(\sigma_{\mathrm{as}}^{2}\right)$, and residual variance $\left(\sigma_{\mathrm{e}}^{2}\right)$, expressed as the percentage of phenotypic variance (defined as the sum of variance components in the model)

\begin{tabular}{lcccc}
\hline Trait & $\sigma_{\mathrm{a}}^{2}$ & $\sigma_{\mathrm{as}}^{2}$ & $\sigma_{\mathrm{e}}^{2}$ & $\mathrm{~h}^{2}$ \\
\hline FF & 0.67 & 0.19 & 0.14 & 0.67 \\
TA & 0.17 & 0.19 & 0.64 & 0.17 \\
Shape & 0.58 & 0.29 & 0.13 & 0.58 \\
RUS & 0.54 & 0.27 & 0.19 & 0.54 \\
SCU & 0.61 & 0.00 & 0.39 & 0.61 \\
OCOL & 0.86 & 0.00 & 0.14 & 0.86 \\
BIT & 0.39 & 0.06 & 0.54 & 0.39 \\
\hline
\end{tabular}

Abbreviations: FF, fruit firmness; TA, titratable acidity; RUS, russet; $S C U$, scuffing; OCOL, skin over-colour; BIT, bitterness. Estimate of heritability $\left(h^{2}\right)$ is also shown for various traits (FF and TA; shape-RUS, SCU, OCOL and BIT).

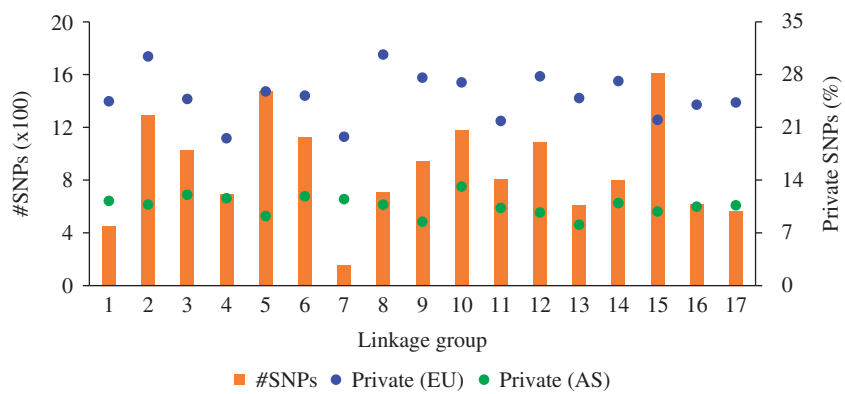

Figure 1. Distribution of retained SNP markers across different linkage groups. The frequency (\%) of SNP markers private to the European (EU) and Asian (AS) species is shown, for each linkage group ( $x$ axis), on the secondary $y$ axis.

all other traits (Table 1$)$. The skin over-colour was the most highly heritable trait $\left(h^{2}=0.86\right)$.

\section{GBS and SNP calling}

Using 'site coverage' of 0.1, a total of 597032 and 395710 SNP markers were detected on $P$. communis and $P$. $\times$ bretschneideri genomes, respectively. Using a higher site coverage (0.9) drastically reduced the number of detected markers to 54121 on 1979 P. communis scaffolds and to 47821 markers on 776 $P . \times$ bretschneideri scaffolds. The next step was to assign these 2755 (=1979+776) scaffolds to LGs according to the 'Suli'32 and 'Bartlett' ${ }^{33}$ maps. If a scaffold had all markers mapped onto one LG in the corresponding map, the scaffold was assigned to that particular LG (and classified as type I scaffold). Following this approach, 597 type I scaffolds (out of 2755) were assigned to a particular LG. Scaffolds on the same LG were ordered by their map position (cM) in the genetic maps. SNP loci detected on these type I scaffolds were kept for further quality checks.

The median read depth per SNP locus was 118, and SNP loci with read depth $<8$ or $>1000$ were removed. After some additional filtering criteria (number of alleles, MAF and missing data frequency), the number of retained SNP markers varied from 157 (on LG7) to 1,610 (on LG15), with a total of 15146 (Figure 1). About $9 \%$ of these SNP were common to those mapped previously to the linkage maps of $P . \times$ bretschneideri and $P$. communis. Across all 214 accessions, the MAF at various SNP loci varied between 0.025 and 0.50 , with an average of 0.18 . However, about 25 and $11 \%$ of all markers were found fixed in the European ( $P$. communis) and Asian (all species other than $P$. communis) pear groups, respectively, while all SNP loci were heterozygous in the hybrid group. The number of fixed loci was fairly consistent between LGs, that is, ranging from $20 \%$ to $30 \%$ and $8 \%$ to $13 \%$ for the European and Asian species, respectively. The difference between the Asian and European pears, in terms of the number of private alleles, was largest (139 vs. 393, respectively) on LG2 and smallest (18 vs. 31, respectively) on LG7 (Figure 1). Different allele frequencies in different genetic groups can result in most loci out of Hardy-Weinberg equilibrium. Thus, SNP markers departing from $\mathrm{H}-\mathrm{W}$ equilibrium were not discarded, however, population structure was taken into account for further analyses.

\section{Population differentiation, structure and LD}

The first principal component (PC1) grouped the accessions belonging to Asian and European species in two non-overlapping clusters. Hybrids among the Asian species clustered within their parental group, but the hybrids between Asian and European pears resided in between the two main clusters depending on the degree of introgression from the parental species (Figure 2a). PC2 revealed the variability within each of the three genetic groups (Asian, European and Hybrid). A break-away group of a few accessions of $P . \times$ bretschneideri and $P$. pyrifolia formed a subgroup within the Asian cluster (top left-hand corner of Figure 2a) where representatives of some of the other Asian species also co-located. The genome-wide gene diversity $\left(H_{s}\right)$ was 0.25 for the Hybrid group, which is slightly higher than the European $(0.21)$ and Asian (0.20) pears. The overall observed heterozygosity $\left(H_{o}\right)$ was very similar for the Asian (0.18), European (0.20) and hybrid (0.18) genetic group.

The genome-level population-differentiation statistic $\left(F_{s t}\right)$ between Asian and European pears was estimated at 0.44, indicating a very strong population differentiation. The overall estimated $F_{s t}$ between the European and Hybrid groups was 0.21 , which is twice that between the Asian and Hybrid pears (0.10). The distribution of $F_{s t}$ values between pairs of genetic groups is shown in Supplementary Figure S2. $F_{I S}$, which measures inbreeding, was highest in the Hybrid group (0.28) followed by the Asian (0.18) and European pears (0.05). High $F_{I S}$ values at the group level also indicate a cryptic population structure within each group, which is also supported by the branching patterns of accessions within each major group on a phylogenetic tree (Figure 2b). The NJ tree reflected larger genetic distances between the European and Asian accessions, whereas hybrids were generally in the middle of the two genetic groups (Figure $2 b$ ).

Admixture analyses were run for population structure models assuming $K$ (the number of clusters) $=1$ to 4 . The likelihood of the posterior density ( $\mathrm{Ln}$ (PD) value changed very little for $K>2$ (Figure $2 \mathrm{c}$ ) and the $\Delta K$ statistic, designed to identify the most relevant number of clusters, was the highest for $K=2$. The mean estimated membership of the Asian accessions to the two clusters, namely Asian pears and European pear, was $96 \%$ and $4 \%$, respectively (Figure $2 \mathrm{~d}$ ); whereas the membership of the European accessions to these clusters was $1 \%$ and $99 \%$ respectively. The interspecific hybrids displayed an average 55 and 45\% introgression from the Asian and European species, respectively (Figure 2d). All available accessions (214) were used for calculating the LD statistics $\left(r^{2}\right)$ between pairs of markers on the same scaffold. The average within-scaffolds LD $\left(r^{2}\right)$ values were 0.20 , $0.10,0.07$ and 0.05 for markers separated by 10, 100, 500 and $1000 \mathrm{~kb}$ distance, respectively (Figure 3).

Evidence of selection and genotype-phenotype association A total of 24 SNP loci located on LGs 2, 3, 6, 10, 12, 14, 15 and 16 displayed substantial selection signatures (log10 Bayes Factor $>0.5$ ), consistent with a model of directional selection at the associated SNP marker or other closely linked genes (Figure 4; Table 2). None of the significant loci displayed balancing or puri- 
a

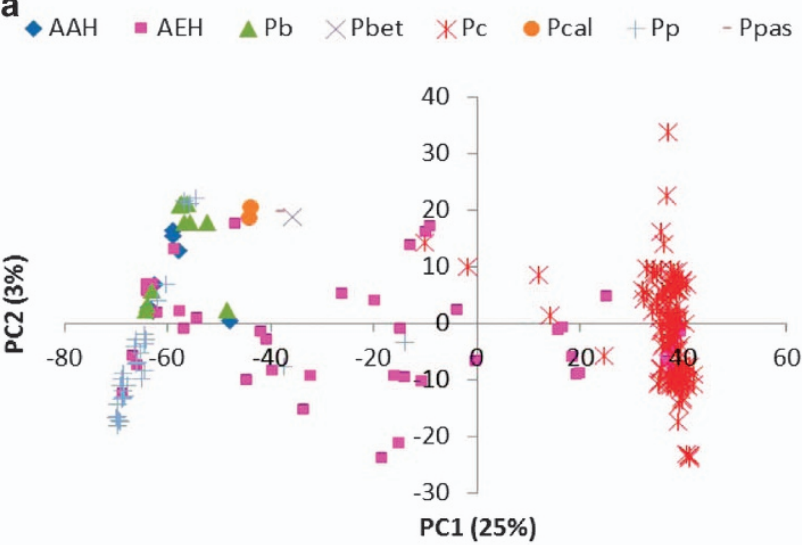

C

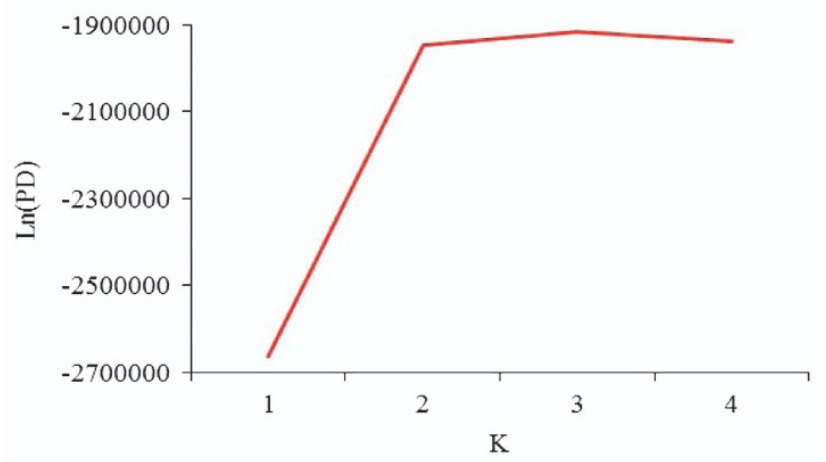

b

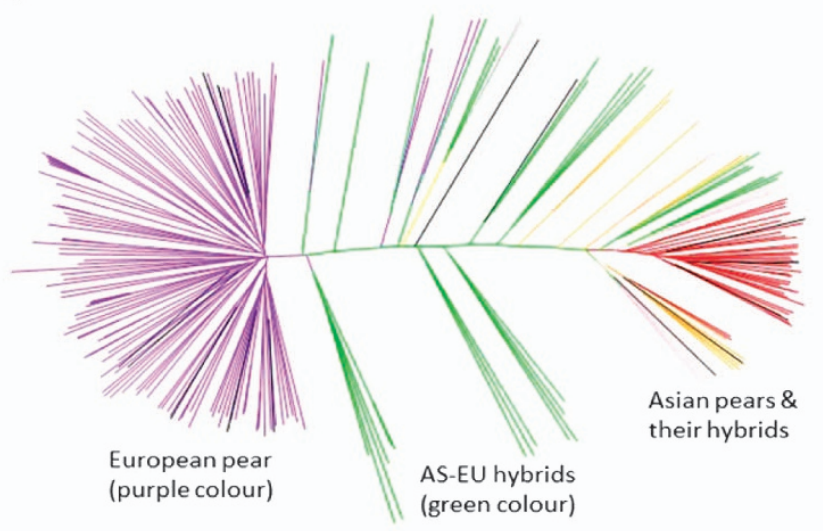

d

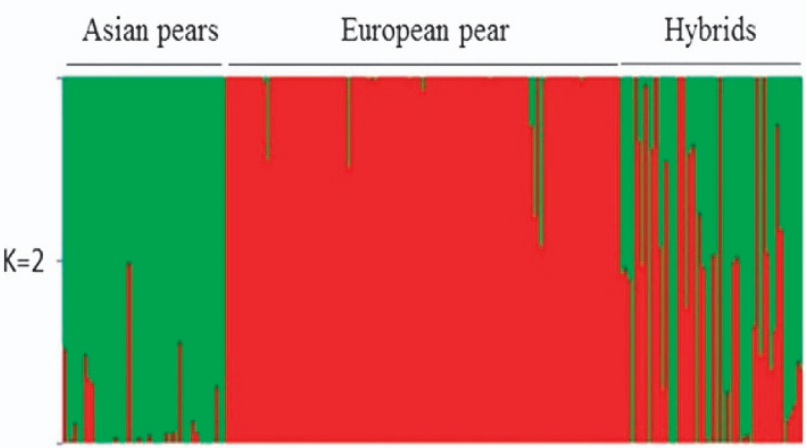

Figure 2. Population structure analysis using principal component (a), neighbour joining (b) and Bayesian clustering (c, d). AAH: Asian-Asian hybrid; AEH: Asian-European hybrid; Pb: P. × bretschneideri; Pbet: $P$. betulaefolia; Pc: $P$. communis; Pcal: P. calleryana; Pp: P. pyrifolia; Ppas: $P$. pashia. (c): The likelihood of the posterior density $(\operatorname{Ln}(P D)$ is shown for various numbers of clusters $(K)$. (d): The mean estimated membership probability ( $y$ axis) of each accession ( $x$ axis) to the two clusters $(K=2)$.

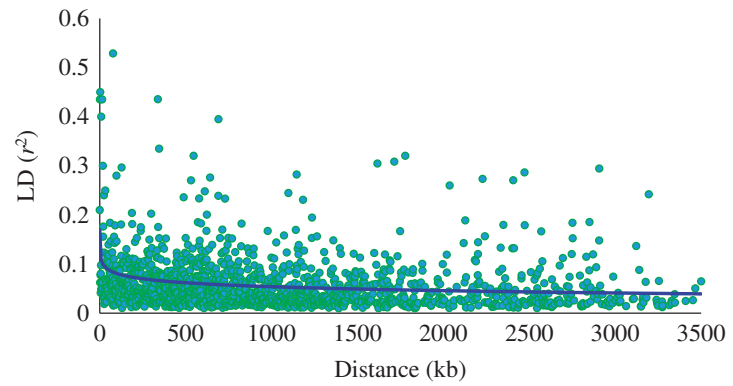

Figure 3. Average (across all scaffolds) linkage disequilibrium $\left(L D ; r^{2}\right)$ values. Decay of LD with distance (kilobase) was estimated from a logarithmic trend line (blue colour).

fying selection. A separate analysis of only the Asian and European groups did not identify any new SNP in addition to those identified from analysis of all three genetic groups together, suggesting that selection signatures in our study are dominated by allele frequency differences between parental species and their hybrids.

For some fruit quality traits, phenotypic variation was significantly correlated with genetic differentiation (that is, $\mathrm{PC} 1$ scores) between the three groups. The highest correlation (0.62) was observed between fruit firmness and PC1 scores (Figure 5). GWA showed that the majority of individual markers explained only a small proportion of phenotypic variation $(\approx 0.5 \%)$, while the largest-effect SNP explained $8 \%, 8 \%, 6 \%, 10 \%, 9 \%$ and $6 \%$ of the phenotypic variation for firmness, TA, shape, russet, scuffing and skin over-colour, respectively (Table 3 and Supplementary Figure S3). Our study also

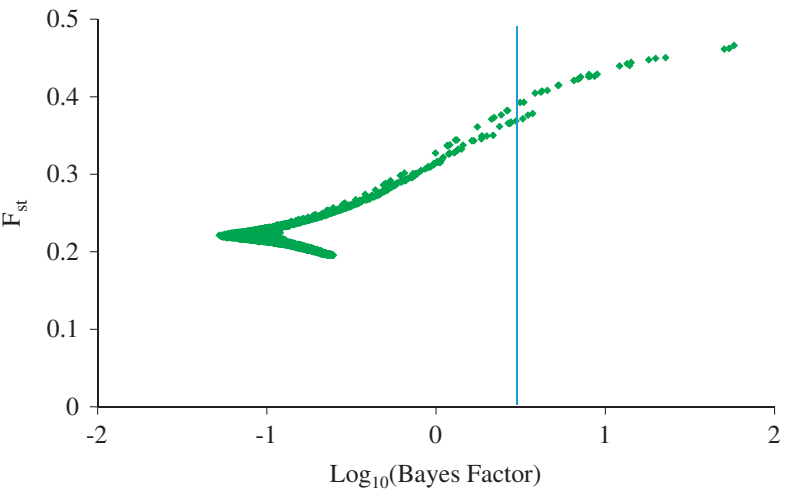

Figure 4. Genome-wide scans for identification of $\mathrm{F}_{\mathrm{st}}$ outlier loci potentially subject to differential selection. Shown are Log transformed Bayes factors (BF) and locus-specific $F_{\text {st }}$ from BayeScan. Vertical line mark $\log _{10}(\mathrm{BF})$ of 0.5 corresponding to posterior probability of locus effects of 0.95 .

found markers, located on LGs 3, 5, 9 and 17, each accounting for about $5 \%$ of variation in fruit firmness. The three markers most strongly associated with the presence of red skin colour were located on LGs 9, 14 and 5, each explaining about 4-6\% of the phenotypic variation (Supplementary Figure S3 and Table 3). Quantile-quantile (QQ) plots, comparing GWA probability with that expected under the null hypothesis of no association, are shown for each trait in Supplementary Figure S4. There was no highly significant SNP associated with skin bitterness. 
Table 2. Single-nucleotide polymorphic (SNP) markers displaying substantial signature of selection (log10 Bayes Factor $>0.5)$

\begin{tabular}{lcccll}
\hline SNP & Scaffold & Position (basepair) & Linkage group & Reference genome & Gene identity (annotation) \\
\hline X2_snp1794 & 4 & 361402 & 2 & $P . \times$ bretschneideri & Unknown \\
X2_snp1979 & 4 & 1426690 & 2 & $P . \times$ bretschneideri & Unknown \\
X2_snp1992 & 4 & 1426910 & 2 & $P . \times$ bretschneideri & Unknown \\
X2_snp906 & 183 & 233831 & 2 & $P . \times$ bretschneideri & Unknown \\
X2_snp911 & 183 & 233846 & 2 & $P . \times$ bretschneideri & Unknown \\
X2_snp936 & 183 & 530882 & 2 & $P . \times$ bretschneideri & Unknown \\
X3_snp366 & 115 & 494820 & 3 & $P . \times$ bretschneideri & Unknown \\
X6_snp1827 & 67 & 516082 & 6 & $P . \times$ bretschneideri & Unknown \\
X6_snp1580 & 506 & 169138 & 6 & $P . \times$ bretschneideri & Unknown \\
X6_snp1608 & 506 & 217289 & 6 & $P . \times$ bretschneideri & Pbr029838.1 (unknown) \\
X6_snp1618 & 506 & 217327 & 6 & $P . \times$ bretschneideri & Pbr029838.1 (unknown) \\
X6_snp2558 & 55014 & 724 & 6 & $P$. communis & Unknown \\
X10_snp1622 & 667 & 202140 & 10 & $P . \times$ bretschneideri & Pbr035011.1 (protein.systhesis.ribosomal) \\
X10_snp1628 & 667 & 202177 & 10 & $P . \times$ bretschneideri & Pbr035011.1 (protein.systhesis.ribosomal) \\
X10_snp1639 & 667 & 213771 & 10 & $P . \times$ bretschneideri & Unknown \\
X10_snp1667 & 667 & 235820 & 10 & $P . \times$ bretschneideri & Pbr035014.1 (unknown) \\
X12_snp1877 & 365 & 232149 & 12 & $P . \times$ bretschneideri & Unknown \\
X12_snp2084 & 500 & 245042 & 12 & $P . \times$ bretschneideri & Unknown \\
X14_snp1535 & 53856 & 570 & 14 & $P$. communis & Unknown \\
X15_snp125 & 111 & 401240 & 15 & $P . \times$ bretschneideri & Unknown \\
X16_snp57 & 102 & 161133 & 16 & $P . \times$ bretschneideri & Unknown \\
X16_snp67 & 102 & 161284 & 16 & $P . \times$ bretschneideri & Unknown \\
X16_snp275 & 185 & 371007 & 16 & $P . \times$ bretschneideri & Pbr011833.1 (RNA.regulation of transcription.PHD finger \\
& & & & & transcription factor) \\
X16_snp283 & 185 & 371113 & 16 & $P . \times$ bretschneideri & Pbr011833.1 (RNA.regulation of transcription.PHD finger \\
& & & & & transcription factor) \\
\hline
\end{tabular}
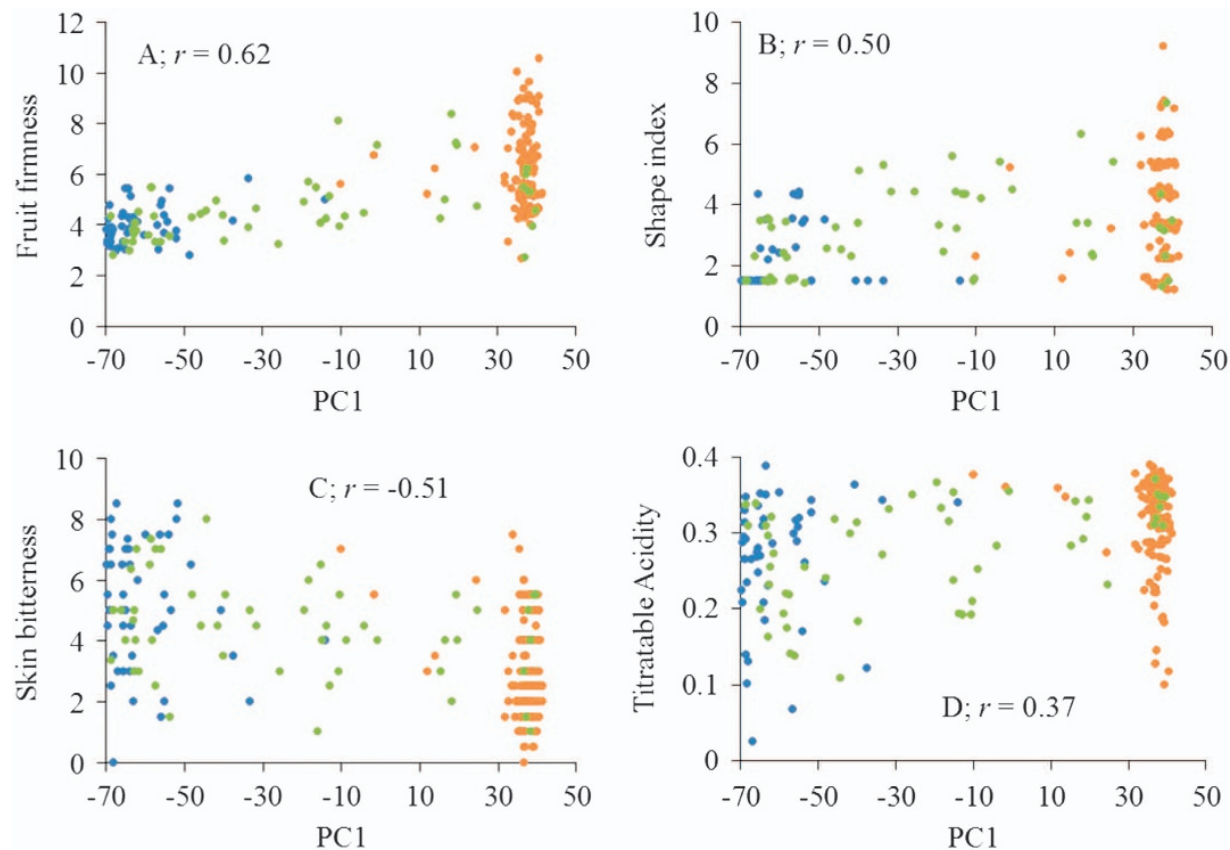

Figure 5. Genotype-phenotype correlation ( $r$ ). $X$ axis: first principal component (PC1) of genome-wide marker scores of accessions; $Y$ axis: phenotypes of Asian (blue), Hybrids (green) and European (orange) accessions.

The frequency of alleles at the largest-effect SNP loci displayed large differences among the three genetic groups (Table 3). For example, the alleles associated with firmness and over-colour at loci S71.0_485233 and S155.0_757109, respectively, were only present in European accessions. Similarly, the alleles associated with TA, russet, and scuffing at loci S895.0_79244, S398.0_140201 and S149.0_728797, respectively, were only present in Asian accessions. The allele associated with fruit shape was present in both European and Asian accessions. The flanking DNA sequences of the largest-effect SNP loci are provided in Supplementary Figure S5.

\section{DISCUSSION}

Species differentiation and genetic diversity

GBS data offers the promise of revealing complex demographic scenarios, and an assessment of gene flow and introgression effects on genetic diversity. The GBS approach implemented in 
Table 3. Single-nucleotide polymorphic (SNP) markers with the largest effect on various traits

\begin{tabular}{|c|c|c|c|c|c|c|c|c|c|c|}
\hline \multirow[t]{2}{*}{ Trait } & \multirow[t]{2}{*}{ Heritability (\%) } & \multicolumn{3}{|c|}{ SNP location } & \multicolumn{3}{|c|}{ Minor allele frequency } & \multicolumn{3}{|c|}{ Significance } \\
\hline & & Linkage group & Scaffold & Position (basepair) & Asian & European & Hybrid & P-value & $F D R$ & $\mathrm{R}^{2}(\%)$ \\
\hline TA & 17 & 2 & S895.0 & 79244 & 0.13 & 0 & 0.11 & $1 \times 10^{-5}$ & 0.165 & 8 \\
\hline Shape & 58 & 13 & S83.0 & 565142 & 0.09 & 0.41 & 0.13 & $1 \times 10^{-5}$ & 0.197 & 6 \\
\hline RUS & 54 & 8 & S398.0 & 140201 & 0.22 & 0 & 0.03 & $1 \times 10^{-6}$ & 0.021 & 10 \\
\hline SCU & 61 & 15 & S149.0 & 728797 & 0.17 & 0 & 0.02 & $8 \times 10^{-6}$ & 0.098 & 9 \\
\hline
\end{tabular}

our study revealed a large number of alleles that were private to either of the two major species groups (European and Asian), which further suggested the suitability of GBS for biodiversity conservation of Pyrus spp. and identification of fine-scale introgressions in interspecific hybrids. Private alleles of some Asian pear species were reported earlier using limited numbers of SSR markers, ${ }^{42}$ but our study provides a much better overview of genome-wide regions harbouring private alleles of European and Asian pears. Geographic isolation of European and Asian pears would suggest independent evolution of these species and thus it is not surprising to find a large number of private alleles in the respective populations. The reproductive isolation of populations is thought to be an initial step towards speciation. ${ }^{43}$ Our findings of adaptation and/or speciation-associated SNP markers are only preliminary, which upon further validation could help in the better management and conservation of genetic resources of European and Asian pears.

The admixed nature of the hybrid group was reflected in its relatively higher gene diversity $\left(H_{s}\right)$ than the ancestral species. The genome-wide inbreeding coefficient $\left(F_{I S}\right)$ of the hybrid group was the highest (0.28), followed by the Asian (0.18) and European (0.05) group of accessions. These results suggested that each genetic group deviated from panmixia, which is consistent with a common practice of assortative mating in pear breeding programmes. $F_{I S}$ values were reported to vary considerably $(0.06-0.18)$ in wild populations of $P$. ussuriensis. ${ }^{44}$ On the basis of 135 SSR markers, Liu et al. ${ }^{7}$ reported an average $F_{I S}$ value of 0.23 across accessions of Asian and European pears. These results indicate somewhat higher $\mathrm{F}_{\mathrm{IS}}$ coefficients of cultivated/advanced accessions compared with wild populations.

Genetic differentiation $\left(\mathrm{F}_{\mathrm{st}}\right)$ between hybrids and European pear was twice of that between hybrids and Asian pears ( 0.21 vs 0.10 ). A key focus of our interspecific breeding programme is to introgress fruit quality traits (for example, crisp textures, resistance to scuffing, spur bearing, long shelf life) from the Asian gene pool into hybrid cultivars, which could partly explain relatively lower $F_{s t}$ between these two groups. Introgression sites are indicated where $\mathrm{F}_{\mathrm{st}}$ values approach zero, that is, where there is no divergence between the regions of hybrid and their ancestral species. ${ }^{45}$ The number of introgression events from the Asian gene pool were higher than the European gene pool, and these events were evident on most LGs (Supplementary Figure S2). The percentage of admixed ancestry of the hybrid group in the Asian gene pool was $55 \%$ (compared with $45 \%$ in the European group), which supports relatively lower genome-wide $F_{s t}$ between these two genetic groups. Understanding the precise position and size of introgressions will help develop molecular markers to reduce linkage drag from donor species. ${ }^{46,47}$
Divergence between the European and Asian species illustrated substantial variation $\left(F_{\text {st }}\right.$ ranging between 0 and 0.99 ) across the whole genome, which is supported by their geographic isolation. $F_{\text {st }}$ values close to 1 suggest reproductive isolation and a high inter-group divergence relative to intra-group diversity. ${ }^{45,48}$ Genome-wide overall estimated $F_{s t}$ between the Asian and European pears was 0.44 , which is much higher than earlier (cf. 0.15 ) reports. ${ }^{7,49}$ Being based on only a small number $(<150)$ of markers, earlier studies could have underestimated the genomewide differentiation. Geographic isolation, local adaptation and population-specific directional selection could have accentuated population differentiation, thus resulting in high observed $F_{s t}$ in our study. Allele frequency differences between parental species and artificial hybrids (Table 3 ) would amount to artificial selection.

\section{Population structure}

Bayesian model-based clustering was conducted to estimate the population structure of 214 Pyrus accessions. The most relevant number of clusters was found to be 2 (Asian and European). The membership coefficient of the European and Asian pears to these two main clusters (0.99 and 0.96 , respectively) suggested a minimal gene flow between the two groups-probably because of their geographic isolation. This low level of gene flow is also a primary cause of high genetic differentiation $F_{s t}$. The average membership coefficient of hybrids to the Asian and European clusters was 0.55 and 0.45 , respectively, but a substantial variation in their membership coefficients was also observed. A small number $(<10)$ of first-generation European-Asian hybrids displayed a high membership coefficient (close to 1.0) of either the Asian or European cluster, suggesting extreme cases of apparent segregation distortion. Distorted segregation, generally observed in interspecific hybrids, could be because of high divergence between parental genome sequences-leading to DNA mismatches during meiotic recombination, which can in turn disrupt meiotic crossovers and accurate chromosome segregation. 47,50

Some $P$. communis cultivars, namely 'Tosca', 'Tenn', 'Moders', 'Florida Home' and 'Jupp' displayed introgression from Asian pears (Figure 2d), while a $P$. $\times$ bretschneideri accession ('Qiyuesu') and a $P$. pyrifolia accession ('Hokusei') displayed membership coefficients of 0.15 and 0.27 , respectively, of the European cluster. One accession (P02) supposedly derived from a cross between $P$. pyrifolia cultivars ('Nijisseiki' $\times$ 'Kosui') was placed half-way between the Asian and European clusters (Figures 2a and d). These results suggested some discrepancy with their documented ancestry. In our study, we had two open-pollinated (OP) seedlings representing $P$. calleryana, and one seedling each of $P$. pashia and $P$. betulaefolia. These four accessions displayed moderate degree of membership coefficient $(0.17-0.25)$ of the European cluster, 
suggesting that these seedlings could have originated from cross fertilisation with $P$. communis.

First principal component (PC1) cleanly separated $P$. communis from Asian pears, which was further supported by a large number of fixed (or private) alleles for these two gene pools (Figure 2a and Figure 1). There were patterns of subgrouping within the Asian pears cluster. For example, five of the $P$. $\times$ bretschneideri accessions ('Xinyali', 'Yali', 'Tsuli', 'Xuehuali' and 'Pingguoli') grouped separately with three red skin accessions ('Winshan' OP, 'Huobali' OP, and 'Yanshan' OP) of $P$. pyrifolia ancestry. 'Pingguoli' is a red skin cultivar and similar to some other cultivars (for example, 'Yali' and 'Tsuli'), has also been classified as $P$. $\times$ bretschneideri or $P$. pyrifolia in different studies. ${ }^{7,51,52}$ The genomes of $P$. ussuriensis and $P$. pyrifolia are thought to have contributed to the origin of $P . \times$ bretschneideri, so it is not surprising to observe overlapping clustering of accessions of these species. ${ }^{8}$

'Beurré Hardy', which is commonly used as interstock between pear scions and quince rootstocks, was located (top right-hand quadrant Figure 2a) away from the rest of the European pear accessions. A sub-group of $P$. communis accessions was also noticeable (lower right-hand quadrant Figure 2a), which consisted of 'Bartlett' bud mutants, namely 'Max Red Bartlett', 'Red Sensation Bartlett, 'Swiss Bartlett' and 'Jumbo Starks'. These results suggested that the genome-wide DNA profiles (that is, genetic constituent) of these 'Bartlett' sports are near identical despite some mutants having red-skin or very large fruit size. Wang et $a l^{53}$ showed that the coding regions of PCMYB10 were the same between red and green sports of 'Max Red Bartlett', but methylation of the promoter region of PCMYB10 repressed the expression of PCMYB10 and subsequently inhibited the biosynthesis of anthocyanin, which probably caused the formation of a green-skin sport of 'Max Red Bartlett'. Qian et al..$^{54}$ also made similar observations for red and green-skin sports of the pear cultivar 'Zaosu', suggesting that inter-retrotransposon amplified polymorphic (IRAP) markers would be better suited to differentiate between bud sports and their original cultivars. ${ }^{55}$

\section{Trait architecture}

Fruit scuffing was among the highly heritable (0.61) traits in this study. Brewer et al. ${ }^{25}$ also reported a high heritability $\left(h^{2}=0.72\right)$ for scuffing and Saeed et al. ${ }^{56}$ mapped numerous QTLs for scuffing, including those on LGs 2 and 15, which supports results of this study and highlights the complex polygenic architecture of this trait. We identified a large-effect SNP (on LG8) associated with fruit russet skin. In previous studies, a marker associated with fruit russet skin was mapped to LG8. ${ }^{57,58}$ Similarly, Song et al. ${ }^{59}$ showed that the pear fruit russet skin trait is linked to apple SSRs $\mathrm{CH} 01$ c06 and Hi2Ob03 located on apple LG8.

Fruit firmness, which is often a key selection trait in pear cultivar breeding programmes, is a highly heritable trait, ${ }^{60}$ but QTL mapping studies ${ }^{56,58}$ did not identify any large effect QTL for this trait. GWA analysis in this study identified a new QTL for fruit firmness on LG16, and the next best SNP explaining about $5 \%$ of variation in fruit firmness was located on LG15, where two ethylene producing genes (PpACS1 and PpACS2) were identified in an earlier study. ${ }^{61}$ The most significant SNP, explaining $8 \%$ of variation in TA, was located on LG2, which is the same one as that of an earlier study using a bi-parental cross between European and Asian species. ${ }^{62}$ The next best SNP associated with TA was located on LG7 (Supplementary Figure S3), which appears to be a new genomic region that has not been reported before. Most genomic regions found significantly associated with fruit shape index were unique, but a QTL on LG2 could coincide with an earlier reported QTL in a bi-parental family. ${ }^{63}$

Functionality of PCMYB10, which can activate the expression of genes encoding enzymes of the anthocyanin biosynthetic pathway leading to red skin colouration of pear fruit, was verified by transient expression assay. ${ }^{64}$ In a cross between 'Abbé Fétel' and 'Max Red Bartlett', Pierantoni et al. ${ }^{65}$ mapped PCMYB10 on LG9 of both cultivars. Our analysis identified a SNP associated with the red-skin phenotype on LG9, but it is not clear if this SNP resides in close proximity of PCMYB10. Although PCMYB10 appears to play a role in the pigmentation of the pear fruit skin, other MYB genes in combination with sunlight and temperature could also contribute to the phenotypic variation in pear red skin colour variation. For example, Dondini et al. ${ }^{66}$ mapped a major gene associated with red skin colour on LG4 of the 'Max Red Bartlett' map. QTL for red skin colour have also been mapped on $\mathrm{LG}_{16}{ }^{32}$ suggesting a complex polygenic nature of this trait.

GWA is a powerful technique for detecting functional variants based on the association between genome-wide markers and phenotypes caused by LD between markers and causal genes or QTL. The maximum phenotypic variance explained by individual SNP markers was less than $10 \%$ in this study, probably due to faster LD decay in wider germplasm compared with family-based designs. ${ }^{67}$ The extent of LD in a sample of Japanese cultivars, reported by lwata et al., ${ }^{68}$ was almost twice that observed in our study, which could mainly be due to the large diversity and admixture nature of our study population. Due to faster LD decay, GWA in admixed populations is far more challenging than in homogeneous populations and requires a relatively higher marker density and population size. ${ }^{69,70}$

\section{Selection footprint}

Genome scans and $F_{\text {st }}$ outlier approaches can be effective at identifying genes under selection without known phenotypes. Extreme differentiation in allele frequencies between genetic groups or geographic zones as measured by the $F_{\text {st }}$ provide signatures of recent positive selection. ${ }^{23}$ Relevance of admixed populations (for example, interspecific hybrids) along with ancestral breeds/species have been shown as an attractive biological model to study adaptive or directional selection. ${ }^{71}$ Using an admixed population in this study, we identified loci with $\log _{10}$ (Bayes factor) $>0.5$ (corresponding to 'substantial' evidence for selection on the Jeffrey's scale of evidence for Bayes factors) to show evidence of selection between the three genetic groups (European, Asian and Hybrids) of accessions.

Association between genotypes and phenotypes in differentiated populations provides an additional tool for identifying genomic regions that may form the genetic basis for the observed phenotypic diversity. ${ }^{72}$ Significant correlation between PC1 (which cleanly separated three genetic groups; Figure 2a) and fruit phenotypes (Figure 5) supported the hypothesis that populationrestricted artificial selection could have played a role in the observed signature footprints in our study. The fact that alleles influencing certain fruit phenotypes were present in hybrid individuals and absent in one of the ancestral species, suggesting that artificial selection for fruit phenotypes could have played a role in the observed allele frequency differences at these largeeffect SNP loci (Table 3). These results provide molecular evidence that a strong directional selection for fruit quality traits in the interspecific breeding programme ${ }^{4}$ have indeed yielded desired outcomes. Brewer et al. ${ }^{25}$ observed that Asian accessions were the source of tolerance to scuffing in an interspecific hybrid population. As the allele associated with scuffing at the largesteffect SNP locus was not present in the European accessions (Table 3 ), our results suggests selection for tolerance to scuffing in Asian pears.

The selection outlier loci in our study did not co-localise with GWA signals. There are various possibilities for these observations. First, it is likely that outlier loci harbour polymorphisms associated with adaptive traits (for example, phenology, reproduction and tolerance to biotic and abiotic stress tolerance) not measured in this study. For example, LG2 harbours large effect QTL for pear 
fire-blight $^{73,74}$ and polygenic scab resistance. ${ }^{75}$ Second, chromosome-level sequences for the genome of Asian pear $(P . \times$ bretschneideri) and European pear (P. communis) are not yet available, so the genomic coordinates of selection loci and GWA SNP markers are not well defined. Hence, the large-effect SNP identified on LGs 2, 10 and 16 could well be in close proximity of markers from selection scans on these LGs. The number of SNP markers used in this study was sufficient to accurately cluster accessions of different genetic groups. However, an increased SNP density could have helped improve the resolution of differentially selected loci, and improve the concordance between the SNP loci identified from GWA and selection footprint analyses. ${ }^{72}$

\section{CONCLUSIONS}

Selection scans and genotype-phenotype association patterns provided preliminary indication of adaptation and selection footprints. Admixture proportions and genome-wide introgression patterns suggested some extreme cases of segregation distortion in interspecific hybrids derived from crosses between the European and Asian species. As the largest-effect markers only explained $<10 \%$ of phenotypic variation, the genetic architecture of pear fruit phenotypes appears to be complex and polygenic. Findings from our study have important implications for our understanding of independent evolution of Asian and European pears, and for the conservation of allelic diversity under rapidly increasing pressure from changing climatic and economic conditions.

\section{CONFLICT OF INTEREST}

The authors declare no conflict of interest.

\section{ACKNOWLEDGEMENTS}

This research was partly supported by the New Zealand Ministry of Business, Innovation and Employment (MBIE). The PFR Markers \& Mapping Team is thanked for help in collecting leaf samples and DNA extraction. Marlene Aldworth, Chris Morgan, David Anderson and Angela Shirtliff helped in fruit harvest and phenotyping. Thanks to Elena Hilario for providing training and guidance for developing the pear GBS protocol. Isabell Moller and David Chagne are thanked for managing the sequencing contract with Macrogen Inc. Constructive feedback from PFR colleagues Elena Hilario, Gail Timmerman-Vaughan and Richard Volz is thankfully acknowledged.

\section{REFERENCES}

1 Silva GJ, Souza TM, Barbieri RL, Oliveira ACD. Origin, domestication, and dispersing of pear (Pyrus spp.). Adv Agr 2014; 2014: e541097.

2 Zielinski QB, Thompson MM. Speciation in Pyrus: chromosome number and meiotic behaviour. Bot Gaz 1967; 128: 109-112.

3 Bell R. Evaluation of Pyrus germplasm for resistance to pear psylla in the orchard. HortScience 2009; 44: 1176.

4 Brewer LR, Morgan C, Alspach PA, Volz RK, White AG. Interspecific pear breeding for flavour and texture. Acta Hort 2008; 800: 461-468.

5 Brewer LR, Palmer JW. Global pear breeding programmes: goals, trends and progress for new cultivars and new rootstocks. Acta Hort 2010; 909: 105-119.

6 Weir BS, Cockerham CC. Estimating F-statistics for the analysis of population structure. Evolution 1984; 38: 1358-1370.

7 Liu Q, Song Y, Liu L et al. Genetic diversity and population structure of pear (Pyrus spp.) collections revealed by a set of core genome-wide SSR markers. Tree Genet Genomes 2015; 11: 1-22.

8 Teng Y, Tanabe K, Tamura F, Itai A. Genetic relationships of Pyrus species and cultivars native to East Asia revealed by randomly amplified polymorphic DNA markers. J Am Soc Hort Sci 2002; 127: 262-270.

9 Song Y, Fan L, Chen $\mathrm{H}$ et al. Identifying genetic diversity and a preliminary core collection of Pyrus pyrifolia cultivars by a genome-wide set of SSR markers. Sci Hortic 2014; 167: 5-16.

10 Volk GM, Richards CM, Henk AD, Reilley AA, Bassil NV, Postman JD. Diversity of wild Pyrus communis based on microsatellite analyses. J Am Soc Hort Sci 2006; 131: 408-417.
11 Miranda C, Urrestarazu J, Santesteban LG, Royo JB, Urbina V. Genetic diversity and structure in a collection of ancient Spanish pear cultivars assessed by microsatellite markers. J Am Soc Hort Sci 2010; 135: 428-437.

12 Kimura T, Shi YZ, Shoda M et al. Identification of Asian pear varieties by SSR analysis. Breeding Sci 2002; 52: 115-121.

13 Erfani J, Ebadi A, Abdollahi H, Fatahi R. Genetic diversity of some pear cultivars and genotypes using simple sequence repeat (SSR) markers. Plant Mol Biol Rep 2012; 30: 1065-1072.

14 Iketani $\mathrm{H}$, Yamamoto $\mathrm{T}$, Katayama $\mathrm{H}$ et al. Introgression between native and prehistorically naturalized (archaeophytic) wild pear (Pyrus spp.) populations in Northern Tohoku, Northeast Japan. Conserv Genet 2010; 11: 115-126.

15 Shi J, Lee S. A novel random effect model for GWAS meta-analysis and its application to trans-ethnic meta-analysis. Biometrics 2016; 72: 945-954.

16 Estoup A, Jarne P, Cornuet JM. Homoplasy and mutation model at microsatellite loci and their consequences for population genetics analysis. Mol Ecol 2002; 11: 1591-1604.

17 Kalinowski ST. Evolutionary and statistical properties of three genetic distances. Mol Ecol 2002; 11: 1263-1273.

18 O'reilly PT, Canino MF, Bailey KM, Bentzen P. Inverse relationship between $F_{\text {st }}$ and microsatellite polymorphism in the marine fish, walleye pollock (Theragra chalcogramma): implications for resolving weak population structure. Mol Ecol 2004; 13: 1799-1814.

19 Wu J, Wang Z, Shi Z et al. The genome of the pear (Pyrus bretschneideri Rehd.). Genome Res 2013; 23: 396-408.

20 Chagné D, Crowhurst RN, Pindo $M$ et al. The draft genome sequence of European pear (Pyrus communis L.'Bartlett'). PLoS ONE 2014; 9: e92644.

21 Elshire RJ, Glaubitz JC, Sun Q et al. A robust, simple genotyping-by-sequencing (GBS) approach for high diversity species. PLoS ONE 2011; 6: e19379.

22 Deschamps S, Llaca V, May GD. Genotyping-by-sequencing in plants. Biology 2012; 25: 460-483.

23 Akey JM, Zhang G, Zhang K, Jin L, Shriver MD. Interrogating a high-density SNP map for signatures of natural selection. Genome Res 2002; 12: 1805-1814.

24 White AG, Brewer LR. The New Zealand pear breeding project. Acta Hort 2000; 596: 239-242.

25 Brewer LR, Morgan CGT, Alspach PA, Volz RK. Heritability and parental breeding value estimates of abrasion-induced skin discolouration on pear fruit. Acta Hort 2011; 909: 127-135.

26 Van Raden PM. Efficient methods to compute genomic predictions. J Dairy Sci 2008; 91: 4414-4423.

27 Gilmour AR, Cullis BR, Harding SA, Thompson R. ASReml Update: What's new in Release 2.00. VSN Int. Ltd: Hemel Hempstead, UK. 2006.

28 Doyle J, Doyle JL. Genomic plant DNA preparation from fresh tissue-CTAB method. Phytochem Bull 1987; 19: 11-15.

29 van Gurp T. 2011. Deena Bioinformatics. Available at www.deenabio.com; www. deenabio.com/services/gbs-adapters.

30 Bradbury PJ, Zhang Z, Kroon DE, Casstevens TM, Ramdoss Y, Buckler ES. TASSEL: software for association mapping of complex traits in diverse samples. Bioinformatics 2007; 23: 2633-2635.

31 Langmead B, Salzberg SL. Fast gapped-read alignment with Bowtie 2. Nat Methods 2012; 9: 357-359.

$32 \mathrm{Wu}$ J, Li LT, Li M et al. High-density genetic linkage map construction and identification of fruit-related QTLs in pear using SNP and SSR markers. J Exp Bot 2014; 65: 5771-5781.

33 Li L, Deng C, Knaebel M et al. Integrated high-density consensus genetic map of Pyrus and anchoring of the 'Bartlett' v1.0 (P. communis) genome. DNA Res 2017 (in press).

34 Money D, Gardner K, Migicovsky Z, Schwaninger H, Zhong GY, Myles S. Linklmpute: Fast and accurate genotype imputation for nonmodel organisms. G3 2015; 5: 2383-2390.

35 Paradis E, Claude J, Strimmer K. APE: analyses of phylogenetics and evolution in R language. Bioinformatics 2004; 20: 289-290.

36 Pritchard JK, Stephens M, Donnelly P. Inference of population structure using multilocus genotype data. Genetics 2000; 155: 945-959.

37 Mangin B, Siberchicot A, Nicolas S, Doligez A, This P, Cierco-Ayrolles C. Novel measures of linkage disequilibrium that corrects the bias due to population structure and relatedness. Heredity 2012; 108: 285-291.

38 Goudet J. R Package Hierfstat 2014 Available at http://www.r-project.org; https:// CRAN.R-project.org/package $=$ hierfstat.

39 Foll M. BayeScan v2. 1 User Manual. Ecology 2012; 20: 1450-1462.

40 Henderson CR. Application of Linear Models in Animal Breeding. University of Guelph: Guelph, ON, Canada. 1984.

41 Lipka AE, Tian F, Wang $Q$ et al. GAPIT: genome association and prediction integrated tool. Bioinformatics 2012; 28: 2397-2399.

42 Kato S, Imai A, Rie N, Mukai Y. Population genetic structure in a threatened tree, Pyrus calleryana var. dimorphophylla revealed by chloroplast DNA and nuclear SSR locus polymorphisms. Conserv Genet 2013; 14: 983-996. 
43 Capy P, Veuille M, Paillette M, Jallon JM, Vouidibio J, David JR. Sexual isolation of genetically differentiated sympatric populations of Drosophila melanogaster in Brazzaville, Congo: the first step towards speciation? Heredity 2000; 84: 468-475.

44 Iketani H, Yamamoto T, Katayama H, Uematsu C, Mase N, Sato Y. Introgression between native and prehistorically naturalized (archaeophytic) wild pear (Pyrus spp.) populations in Northern Tohoku, Northeast Japan. Conserv Genet 2010; 11: 115-126.

45 Neafsey DE, Barker BM, Sharpton TJ et al. Population genomic sequencing of Coccidioides fungi reveals recent hybridization and transposon control. Genome Res 2010; 20: 938-946.

46 Lin T, Zhu G, Zhang J et al. Genomic analyses provide insights into the history of tomato breeding. Nat Genet 2014; 46: 1220-1226.

47 Montanari S, Brewer L, Lamberts R et al. Genome mapping of postzygotic hybrid necrosis in an interspecific pear population. Hort Res 2016; 3: 15064.

48 Nosil P, Parchman TL, Feder JL, Gompert Z. Do highly divergent loci reside in genomic regions affecting reproductive isolation? A test using next-generation sequence data in Timema stick insects. BMC Evol Bio 2012; 12: 1.

49 Bao L, Chen KS, Zhang D, Cao YF, Yamamoto T, Teng YW. Genetic diversity and similarity of pear (Pyrus L.) cultivars native to East Asia revealed by SSR (simple sequence repeat) markers. Genet Resour Crop Evol 2007; 54: 959-971.

50 Maheshwari S, Barbash DA. The genetics of hybrid incompatibilities. Annu Rev Genet 2011; 45: 331-355.

51 Wang P-W, Liu H-Z, Chen Y-Q, Jin X-L, Qu B-H. RAPD analysis of germplasm resources in Pyrus. Acta Hortic Sin 2001; 28: 460-462.

52 Shaheen MA, Essa MA, Sayed RA, El-Aziz YA. Sexual compatibility of LeConte pear cultivar. J Hortic Sci Ornament Plant 2011; 3: 99-105.

53 Wang Z, Meng D, Wang A et al. The methylation of the PCMYB10 promoter is associated with green-skinned sport in Max Red Bartlett pear. Plant Physiol 2013; 62: 885-896.

54 Qian M, Sun Y, Allan AC, Teng Y, Zhang D. The red sport of 'Zaosu' pear and its red-striped pigmentation pattern are associated with demethylation of the PyMYB10 promoter. Phytochemistry 2014; 107: 16-23.

55 Sun J, Yin H, Li L et al. Evaluation of new IRAP markers of pear and their potential application in differentiating bud sports and other Rosaceae species. Tree Genet Genomes 2015; 11: 1-3.

56 Saeed M, Brewer L, Johnston J et al. Genetic, metabolite and developmental determinism of fruit friction discolouration in pear. BMC Plant Biol 2014; $14: 1$.

57 Inoue $E$, Kasumi M, Sakuma F, Anzai $H$, Amano K, Hara $H$. Identification of RAPD marker linked to fruit skin colour in Japanese pear (Pyrus pyrifolia Nakai). Sci Hortic 2006; 107: 254-258.

58 Yamamoto T, Terakami S, Takada N et al. Identification of QTLs controlling harvest time and fruit skin colour in Japanese pear (Pyrus pyrifolia Nakai). Breeding Sci 2014; 64: 351-361.

59 Song W, Wang $\mathrm{CH}$, Tian YK, Tian W, Yin H. SSR molecular markers linked to the fruit russet skin of pear. Acta Hortic Sin 2010; 37: 1325-1328.

60 Shin IS, Shin YU, Hwang HS. Heritability of fruit characters of interspecific hybrids between Pyrus pyrifolia and P. ussuriensis or P. breschneideri. Acta Hort 2007; 800: 535-540.

61 Itai A, Kotaki T, Tanabe K, Tamura F, Kawaguchi D, Fukuda M. Rapid identification of 1-aminocyclopropane-1-carboxylate (ACC) synthase genotypes in cultivars of Japanese pear (Pyrus pyrifolia Nakai) using CAPS markers. Theor Appl Genet 2003; 106: $1266-1272$
62 Liu JY, Cui HR, Wand L et al. Analysis of pear fruit acid/low-acid trait by SSR marker. J Fruit Science 2011; 3: 004.

63 Zhang RP, Wu J, Li XG et al. An AFLP, SRAP, and SSR genetic linkage map and identification of QTLs for fruit traits in pear (Pyrus L.). Plant Mol Biol Rep 2013; 31: 678-687.

64 Lin-Wang K, Bolitho K, Grafton K et al. An R2R3 MYB transcription factor associated with regulation of the anthocyanin biosynthetic pathway in Rosaceae. BMC Plant Biol 2010; 10: 1.

65 Pierantoni L, Dondini L, De Franceschi P, Musacchi S, Winkel BS, Sansavini S. Mapping of an anthocyanin-regulating MYB transcription factor and its expression in red and green pear, Pyrus communis. Plant Physiol Bioch 2010; 48: 1020-1026.

66 Dondini L, Pierantoni L, Ancarani V et al. The inheritance of the red colour character in European pear (Pyrus communis) and its map position in the mutated cultivar 'Max Red Bartlett'. Plant Breeding 2008; 127: 524-526.

67 Kumar S, Raulier P, Chagné D, Whitworth C. Molecular-level and trait-level differentiation between the cultivated apple (Malus $\times$ domestica Borkh.) and its main progenitor Malus sieversii. Plant Genetic Resources 2014; 12: 330-340.

68 Iwata H, Hayashi T, Terakami S, Takada N, Sawamura Y, Yamamoto T. Potential assessment of genome-wide association study and genomic selection in Japanese pear Pyrus pyrifolia. Breeding Sci 2013; 63: 125-140.

69 Risch N, Tang $\mathrm{H}$. Whole genome association studies in admixed populations. Am J Hum Genet 2006; 79: S254.

70 Toosi A, Fernando RL, Dekkers JC, Quaas RL. Genomic selection in admixed and crossbred populations. J Anim Sci 2010; 88: 32.

71 Gautier M, Naves M. Footprints of selection in the ancestral admixture of a New World Creole cattle breed. Mol Ecol 2011; 20: 3128-3143.

72 Evans LM, Slavov GT, Rodgers-Melnick E et al. Population genomics of Populus trichocarpa identifies signatures of selection and adaptive trait associations. Nat Genet 2014; 46: 1089-1096.

73 Dondini L, Pierantoni L, Gaiotti F et al. Identifying QTLs for fire-blight resistance via a European pear (Pyrus communis L.) genetic linkage map. Mol Breeding 2005; 14: 407-418.

74 Le Roux PM, Christen D, Duffy B et al. Redefinition of the map position and validation of a major quantitative trait locus for fire blight resistance of the pear cultivar 'Harrow Sweet'(Pyrus communis L.). Plant Breeding 2012; 131: 656-664.

75 Won K, Bastiaanse H, Kim YK et al. Genetic mapping of polygenic scab (Venturia pirina) resistance in an interspecific pear family. Mol Breed 2014; 34: 2179-2189.

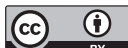

This work is licensed under a Creative Commons Attribution 4.0 International License. The images or other third party material in this article are included in the article's Creative Commons license, unless indicated otherwise in the credit line; if the material is not included under the Creative Commons license, users will need to obtain permission from the license holder to reproduce the material. To view a copy of this license, visit http://creativecommons.org/licenses/ by/4.0/

(c) The Author(s) 2017

Supplementary Information for this article can be found on the Horticulture Research website (http://www.nature.com/hortres) 